\title{
Light Element Analysis Revisited in the Variable Pressure SEM and the nanoSIMS 50 Ion Probe
}

\author{
Brendan J Griffin
}

Centre for Microscopy and Microanalysis, The University of Western Australia, 35 Stirling Highway, Crawley, WA Australia 6009

The ions generated in the electron gas cascade amplification process during imaging with the various gaseous secondary electron detectors (GSED) in VPSEM, have a significant effect on image quality. Attention has focussed on devices that reduce the quantity of such ions, and so to reduce recombinant-based loss of the electron signal, to good effect $[1,2]$.

A second and equally important role of the ions has been briefly reported where these ions have been suggested to take part in a near-surface 'plasma' cleaning process that contains and effectively eliminates hydrocarbon-based surface contamination of the sample. This data revealed an advantage VPSEM has over high vacuum SEM. Despite the long $(900 \mathrm{sec})$ times, the repeat counts $(\mathrm{n}=13)$, at low $\mathrm{E}_{\mathrm{o}}(3 \mathrm{kV})$ and using argon as the chamber gas $\left(0.2\right.$ torr) gave similar results across the $\mathrm{Si}_{3} \mathrm{~N}_{4}$ film on the GaAs substrate (figure 1) [3]. The N:Si data is very consistent and the peak ratio matches modeled (DTSA) data for a $\mathrm{Si}(\mathrm{Li}) \mathrm{EDS}$ with a copolymer window on such a sample.

Nitrogen analysis of diamond has been undertaken in parallel using a nanoSIMS 50 ion probe and initial results were very encouraging. Nitrogen was considered to be present both as aggregates and also more uniformly dispersed in the diamond, the latter vary with growth zones and the nanoSIMS mass image intensities show a strong inverse correlation with cathodoluminescence (figure 2) [4]. The nanoSIMS study of these diamond samples has been continued and several refinements to the initial observations must be made:

(i) the linear arrays seen in the mass 12 (C) images (figure 3(a)) are artifacts that remain constant when the sample is re-oriented; they are now interpreted as implantation effects,

and

(ii) the finer-scale $\mathrm{N}$ aggregates inferred from the mass 26 images (figure 3(b)) do not persist through repeated scans, ie depth, into the sample.

A favoured hypothesis for (ii) is that the fine-scale aggregates are void platelets at the polished surface. They have become $\mathrm{N}$-rich due to their surface exposure and this characteristic disappears at depth or as a consequence of repeated ablation events.

On-going studies are applying the VPSEM EDS approach to these samples in conjunction with further nanoSIMS work. An interesting question has arisen in that do light elements attached or infiltrate even relatively inert sample surfaces during sample preparation? To what degree does this occur is perhaps more correct.

\section{References}

[1] W.R. Knowles et al., Microsc. Microanal. 10 (Suppl 2) (2004) 1060.

[2] J.P. Craven et al., J Microscopy 205 (2002) 96.

[3] B.J. Griffin et al., Institute of Physics Conference Series 165 (2000) 263

[4] B.J. Griffin et al., Microsc. Microanal. 10 (Suppl 2) (2004) 96.

[5] The research was partially funded by the NANO Major National Research Facility 


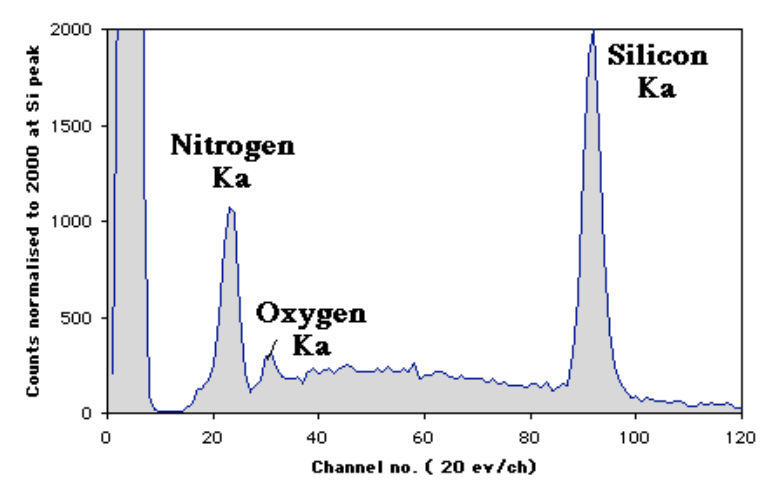

(a)

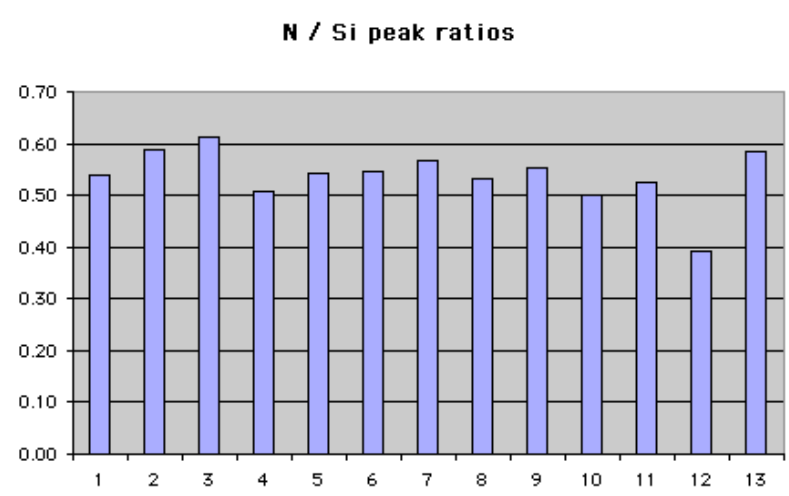

(b)

Figure 1: (a) EDS spectrum of silicon nitride film $(350 \mathrm{~nm})$ on GaAs substrate collected at Eo = $3 \mathrm{kV}$ in argon for 900 seconds in VPSEM, and (b) the N/Si integrated $\mathrm{K} \alpha$ peak ratios for repeat analyses of different areas of the film.

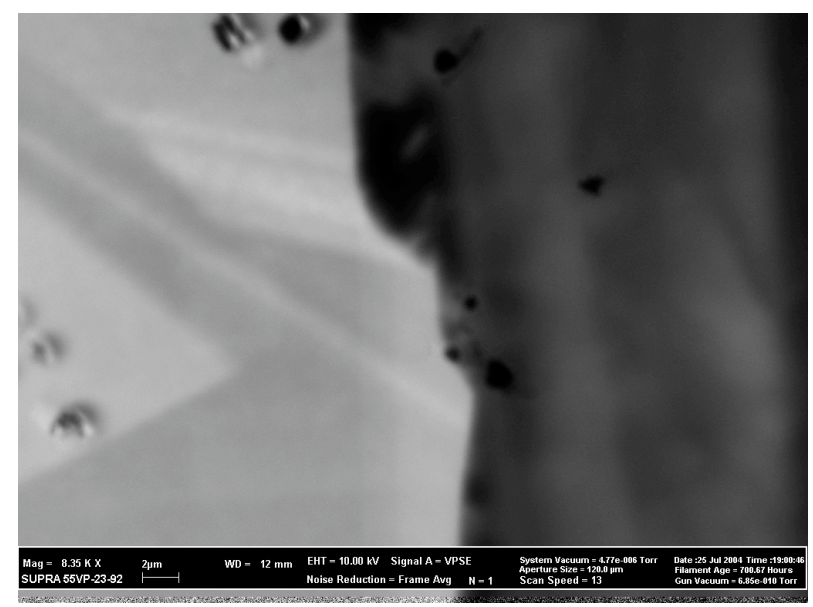

(a)

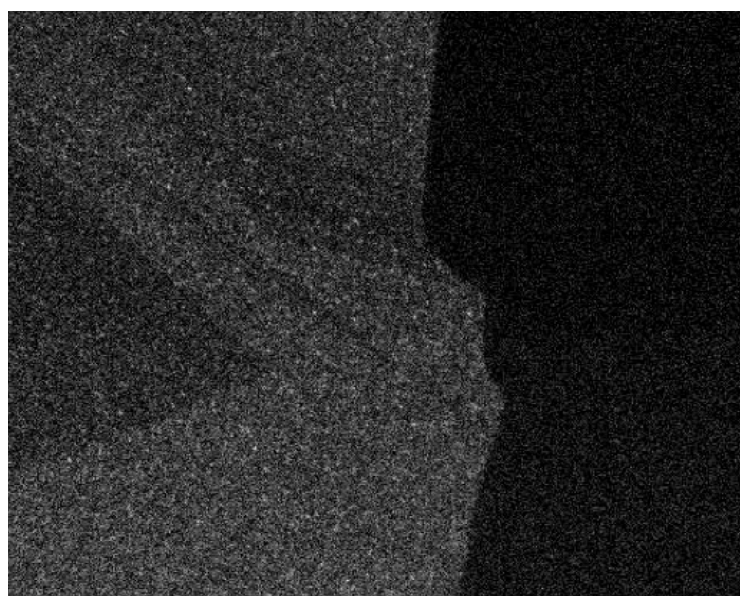

(b)

Figure 2: (a) Cathodoluminescence and (b) mass 26 (C12N14) nanoSIMS ion probe images. The two images show an inverse correlation. Field of view is $15 \mu \mathrm{m}$.

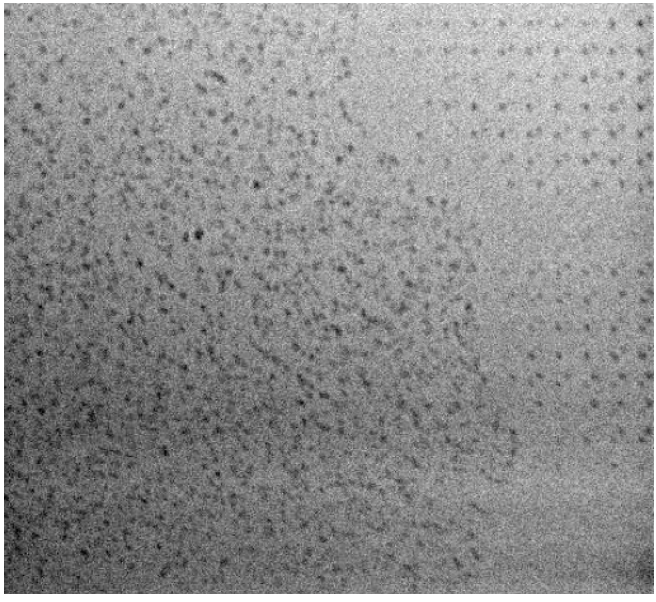

(a)

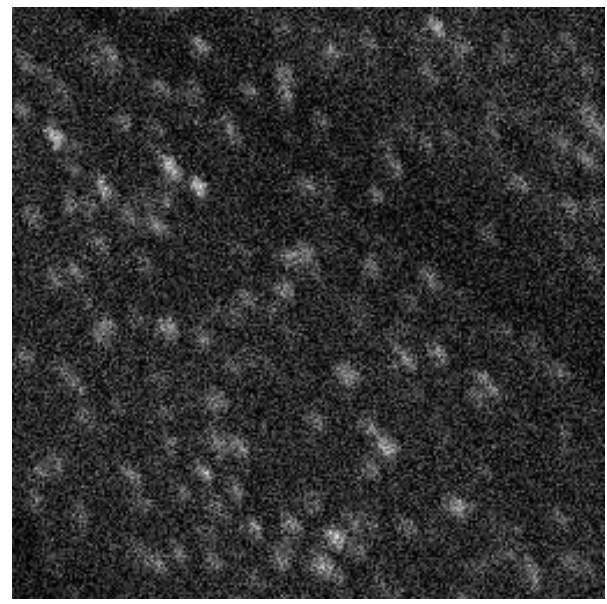

(b)

Figure 3 : (a) a mass 12 (C12) nanoSIMS ion probe image of the same area shown fig 2(b) with an inverse correlation with the mass $26(\mathrm{C} 12 \mathrm{~N} 14)$ data. A higher magnification $(\mathrm{FoV}=5 \mu \mathrm{m})$ mass 26 image (b) reveals the 'linear' $\mathrm{N}$ aggregates present. 